\begin{tabular}{|c|l|}
\hline Title & Nonlinear Carrier Responses in Gold Thin Films Induced by Intense Terahertz Waves \\
\hline Author(s) & Minami, Y asuo; Dao, Thang Duy; Nagao, Tadaaki; Takeda, Jun; Kitajima, Masahiro; Katay ama, Ikufumi \\
\hline Citation & $\begin{array}{l}\text { International Conference on Ultrafast Phenomena 2014 Okinawa Japan 7-11 July 2014 ISBN:1-55752-279-0, paper } \\
\text { O7.Mon.P1.29 }\end{array}$ \\
\hline https:/doi.org/10.1364/NP.2014.07.Mon.P1.29 \\
\hline Issue Date & 2014 \\
\hline Doc URL & http://hdl.handle.net/2115/72281 \\
\hline File Information & UP_07_Mon_P1.pdf \\
\hline
\end{tabular}

Instructions for use 


\title{
Nonlinear Carrier Responses in Gold Thin Films Induced by Intense Terahertz Waves
}

\author{
Yasuo Minami ${ }^{1}$, Thang Duy Dao ${ }^{2,3}$, Tadaaki Nagao ${ }^{2,3}$, \\ Jun Takeda ${ }^{1}$, Masahiro Kitajima ${ }^{1,3,4,5}$, and Ikufumi Katayama ${ }^{1}$ \\ ${ }^{1}$ Department of Physics, Graduate School of Engineering, Yokohama National University, Yokohama 240-8501, Japan \\ ${ }^{2}$ International Center for Materials Nanoarchitectonics, National Institute for Materials Science, Tsukuba 305-0044, Japan \\ CREST, Japan Science and Technology Agency, Kawaguchi 332-0012, Japan \\ ${ }^{4}$ LxRay Co. Ltd., Nishinomiya 663-8172, Japan \\ ${ }^{5}$ Department of Applied Physics, National Defense Academy, Yokosuka 239-8686, Japan \\ E-mail address: minamiyasuo@ynu.ac.jp
}

\begin{abstract}
Terahertz transmittances of the gold thin-films with thicknesses ranging from 1 to $12 \mathrm{~nm}$ were investigated. As terahertz field becomes intense, the transmittance of the terahertz field decreases, suggesting the increase of the carrier density.
\end{abstract}

OCIS codes: (300.6495) Spectroscopy, terahertz; (310.6188) Thin films, Spectral properties; (320.7120) Ultrafast phenomena

\section{Introduction}

Gold $(\mathrm{Au})$ has been extensively studied in the field of microelectronics and bio-sensing, since many attractive electromagnetic properties of Au nanostructures were recently reported; the effective sheet conductivity of Au thin film dramatically decreases with the thickness less than $2 \mathrm{~nm}$ [1]. This suggests that the nano-scale size and morphology play key roles on the electronic properties. In the present study, we investigate the carrier dynamics in $\mathrm{Au}$ thin film with different thicknesses using intense terahertz electric field.

\section{Experiments}

The Au thin film was evaporated on the high resistivity $\mathrm{Si}$ substrate with $\mathrm{Si}(111)-7 \times 7$ surface in the chamber with the ultrahigh vacuum of $\sim 10^{-7} \mathrm{~Pa}$ at room temperature. The crystallization process was confirmed using reflection high-energy electron diffraction, and the polycrystal and flat $\mathrm{Au}$ films were successfully prepared. The thicknesses of the obtained Au films were 1.3, 1.9, 5.8, and $11.5 \mathrm{~nm}$. The details of the experimental setup are reported elsewhere [2,3]. Figure 1(a) shows the SEM image of the specimen with $1.9 \mathrm{~nm}$ thickness, which indicates the flatness of the specimen. The flatness enabled us to investigate the terahertz responses of $\mathrm{Au}$ thin films precisely. The experimental configuration of specimen from the side view is shown in Fig. 1(b).

A Ti:Sapphire amplifier system (repetition rate: 1 $\mathrm{kHz}$, pulse duration: $130 \mathrm{fs}$, center wavelength: $800 \mathrm{~nm}$, pulse energy: $1.6 \mathrm{~mJ} /$ pulse) is employed to generate intense terahertz waves and to detect these terahertz waves. In generating terahertz fields, tilted laser pulses are irradiated onto a $\mathrm{LiNbO}_{3}$ via Cherencov-type phase matching process [4]. The generated terahertz waves are incident on the specimen. Then, the transmitted waves are forwarded to a $0.4 \mathrm{~mm}$-thick $\mathrm{GaP}$ crystal using off-axis parabolic mirrors and finally observed by the electro-optic (EO) sampling. The maximum-terahertz field was 340 $\mathrm{kV} / \mathrm{cm}$. The intensity is tuned by the wire grid polarizers set in front of the specimen. The transmittance of the specimen is obtained by normalizing the transmitted spectrum of the specimen $(\mathrm{Au}$ on $\mathrm{Si}$ ) by that of the reference $(\mathrm{Si})$ (see Fig. 1 (b)). (a)

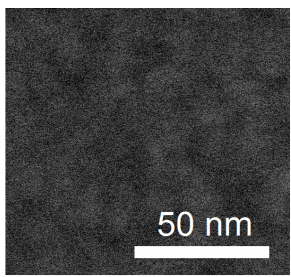

(b)

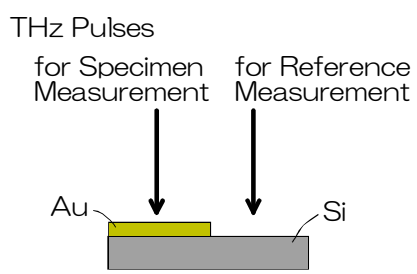

Fig. 1 (a) SEM image of Au thin film surface with $1.9 \mathrm{~nm}$ thickness. (b) Side view of the specimen for measuring terahertz transmittance of the reference and signal.

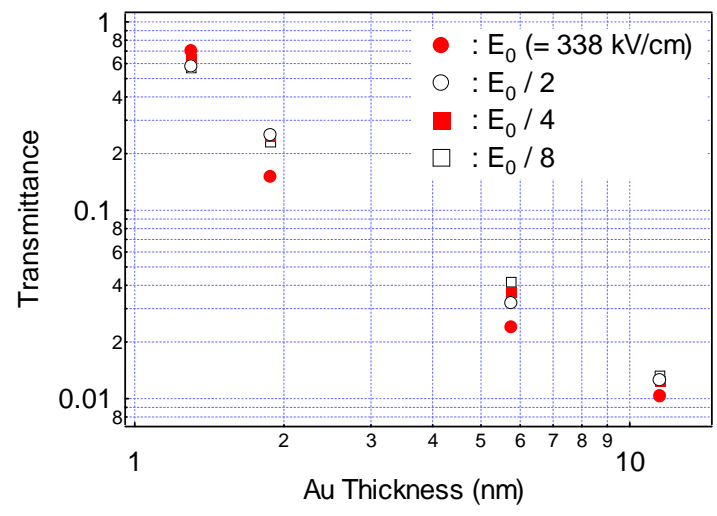

Fig. 2 Transmittance of terahertz waves as a function of the Au film thickness with several terahertz intensities. 
(a)

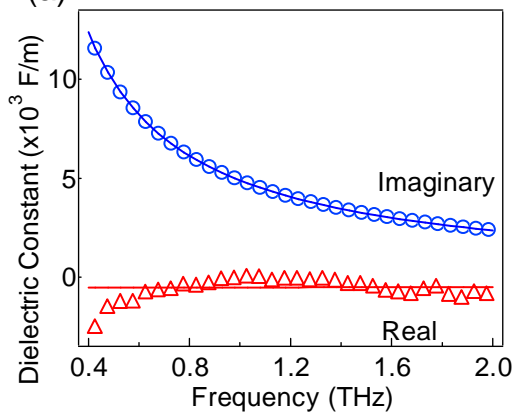

(b)

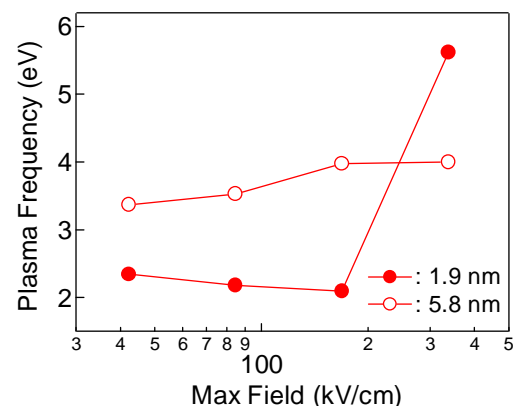

(c)

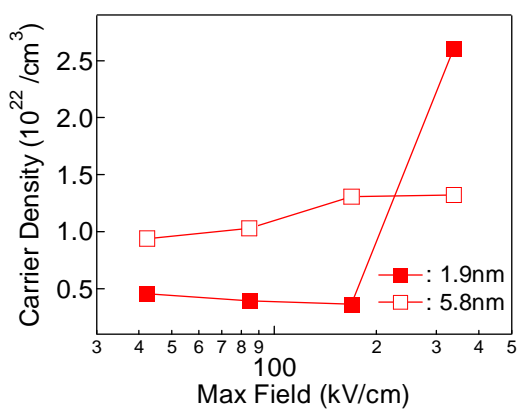

Fig. 3 (a) Complex dielectric constant of gold film with the thickness of $1.9 \mathrm{~nm}$. Solid curves indicate the best-fit to the experimental data obtained by Drude analysis. (b) The estimated plasma frequencies and (c) the carrier densities of the Au films with the thicknesses of 1.9 and 5.8 nm as a function of maximum terahertz electric field.

\section{Results and Discussion}

Figure 2 shows the Au film thickness dependence of the transmittance with different terahertz fields. Except for the case of $1.3 \mathrm{~nm}$-thick film, the transmittance becomes lower with the intense terahertz field illumination. To clarify the reason, Drude analysis was carried out for the complex dielectric constant obtained from the terahertz transmittance. For example, the complex dielectric constant obtained for the Au film with $1.9 \mathrm{~nm}$ thickness is shown in Fig. 3(a). In the Drude model, the plasma frequency of the free electron gas is expressed as

$$
\omega_{\mathrm{p}}=\sqrt{\frac{n e^{2}}{\varepsilon_{0} m^{*}}},
$$

where $n$ is the carrier density, $m^{*}$ is the effective mass of the carrier, $e$ is the elementary charge, and $\varepsilon_{0}$ dielectric constant in vacuum. The plasma frequency of the Au film vs. the maximum electric field is shown in Fig. 3(b). The solid and open circles show the plasma frequencies of the 1.9 and $5.8 \mathrm{~nm}$-thick Au films, respectively. In the weak terahertz field region, the plasma frequencies of $1.9 \mathrm{~nm}$ - and 5.8-nm thick films are similar to those obtained in Ref. [5]. In contrast to the weak terahertz field region, the plasma frequency is strongly enhanced by the intense terahertz field especially in the case of $1.9 \mathrm{~nm}$-thick $\mathrm{Au}$ film. Assuming that the effective mass is constant in Eq. (1), the enhancement of the plasma frequency depends on the increase of the carrier density. The carrier density of the Au film vs. the maximum electric field is shown in Fig. 3(c). The solid and open squares show the carrier densities of the 1.9 and $5.8 \mathrm{~nm}$-thick $\mathrm{Au}$ films, respectively. The carrier density of the $1.9 \mathrm{~nm}$-thick Au film at $338 \mathrm{kV} / \mathrm{cm}$ terahertz field is larger than that of $5.8 \mathrm{~nm}$-thick Au film. This enhancement can be explained by the penetration depth of terahertz electric field. The penetration depth of terahertz wave into the specimen was estimated to be $2 \mathrm{~nm}$ from Fig. 2. Therefore, the carriers in the $1.9 \mathrm{~nm}$-thick film is more susceptible to the influence of terahertz electric field than that in the $5.8 \mathrm{~nm}$-thick film.

In case of the $1.3 \mathrm{~nm}$ thick Au film, the clear enhancement was not observed, probably because of some drastic change in electronic structures taking place between 1 and $2 \mathrm{~nm}$ such as insulator-to-metal transition [1].

\section{Conclusions}

We investigated the transmittance of the $\mathrm{Au}$ thin films with thickness ranging from 1 to $12 \mathrm{~nm}$ by intense terahertz electric fields. In the case of the thickness with $1.9-11.8 \mathrm{~nm}$, the transmittance became lower by the irradiation of intense terahertz fields. The Drude analysis clearly shows that the carrier density is enhanced by the intense terahertz field.

\section{References}

[1] J. J. Tu, C. C. Homes, and M. Strongin, “Optical Properties of Ultrathin Films: Evidence for a Dielectric Anomaly at the Insulator-to-Metal Transition," Phys. Rev. Lett. 90, 017402 (2003).

[2] T. Nagao, J. T. Sadowski, M. Saito, S. Yaginuma, Y. Fujikawa, T. Kogure, T. Ohno, Y. Hasegawa, S. Hasegawa, and T. Sakurai, “Nanofilm Allotrope and Phase Transformation of Ultrathin Bi Film on Si(111)-7×7," Phys. Rev. Lett. 93, 105501 (2004).

[3] S. Yaginuma, K. Nagaoka, T. Nagao, G. Bihlmayer, Y. M. Koroteev, E. V. Chulkov, and T. Nakayama, "Electronic Structure of Ultrathin Bismuth Films with A7 and Black-Phosphorus-like Structures," J. Phys. Soc. Jpn. 77, 014701 (2008).

[4] J. Hebling, G. Almási, I. Z. Kozma, and J. Kuhl, "Velocity matching by pulse front tilting for large area THz-pulse generation," Opt Express 10, 1161 (2002).

[5] J. Siegel, O. Lyutakov, V. Rybka, Z. Kolská, and V. Švorčík, “Properties of gold nanostructures sputtered on glass,” Nanoscale Res. Lett. 6, 96 (2011). 